\title{
Transverse emittance growth in staged laser-wakefield acceleration
}

\author{
T. Mehrling, ${ }^{1}$ J. Grebenyuk, ${ }^{2}$ F. S. Tsung, ${ }^{3}$ K. Floettmann, ${ }^{2}$ and J. Osterhoff ${ }^{1, *}$ \\ ${ }^{1}$ Institut für Experimentalphysik, Universität Hamburg, 22761 Hamburg, Germany \\ ${ }^{2}$ Deutsches Elektronen-Synchrotron DESY, 22607 Hamburg, Germany \\ ${ }^{3}$ Department of Physics \& Astronomy, University of California Los Angeles, Los Angeles, California 90095, USA
}

(Received 30 May 2012; published 8 November 2012)

\begin{abstract}
We present a study on the emittance evolution of electron bunches, externally injected into laser-driven plasma waves using the three-dimensional particle-in-cell (PIC) code OSIRIS. Results show order-ofmagnitude transverse emittance growth during the injection process, if the electron bunch is not matched to its intrinsic betatron motion inside the wakefield. This behavior is supported by analytic theory reproducing the simulation data to a percent level. The length over which the full emittance growth develops is found to be less than or comparable to the typical dimension of a single plasma module in current multistage designs. In addition, the analytic theory enables the quantitative prediction of emittance degradation in two consecutive accelerators coupled by free-drift sections, excluding this as a scheme for effective emittance-growth suppression, and thus suggests the necessity of beam-matching sections between acceleration stages with fundamental implications on the overall design of staged laser-wakefield accelerators.
\end{abstract}

DOI: 10.1103/PhysRevSTAB.15.111303

PACS numbers: 52.38.Kd, 29.20.Ej, 41.75.Jv, 52.40.Mj

\section{INTRODUCTION}

A number of experiments during the past decade [1-7] have confirmed laser-wakefield acceleration of charged particles in plasma as a promising technology candidate for driving compact and brilliant $\mathrm{x}$-ray light sources $[8,9]$ and possibly future particle colliders [10,11]. These plasma waves support extreme field gradients that facilitate $\mathrm{GeV}$ energy gain in centimeter-scale stages [4,5] with the length of a unit and hence the energy gain in a single stage being fundamentally limited by energy depletion of the driving laser pulse [12]. Taking into account current high-end laser technology, acceleration of electron beams to energies beyond the $10 \mathrm{GeV}$ level seems possible only by use of multiple stages in series, i.e. by staging. As a consequence, pivotal beam parameters such as the transverse emittance must be conserved during the transport of preaccelerated electron bunches into the accelerating phase of a subsequent plasma module to allow for high-energy, highquality beams and applications in photon science and at the particle-beam energy frontier.

Matching of particle beams into the focusing fields of plasma accelerators is vital for the conservation of beam quality. Mismatched particle bunches develop a decoherence of particle betatron oscillations and significant beam envelope oscillations [13,14] and thus an increase in

\footnotetext{
*Corresponding author. jens.osterhoff@desy.de

Published by the American Physical Society under the terms of the Creative Commons Attribution 3.0 License. Further distribution of this work must maintain attribution to the author(s) and the published article's title, journal citation, and DOI.
}

emittance. In general, these effects are caused by a nonzero energy spread or a nonzero bunch length. It has been shown in earlier studies that matching of bunch size reduces emittance growth from finite energy spread $[15,16]$, and mitigates energy loss and correlated growth of energy spread due to emission of synchrotron radiation [16]. Emittance growth due to finite bunch length has been seen in some numerical examples and was discussed qualitatively in a recent publication [14].

In this work, we derive a general analytic expression for the emittance growth caused from betatron decoherence as expected by insufficient matching of the beta function (bunch size) or of the alpha function (focusing) and compare it to fully three-dimensional particle-in-cell simulations of finite-length electron bunches with low energy spread, externally injected into a laser-driven plasma wave. In addition we discuss quantitatively the fundamental implications of beam-quality degeneration arising from insufficiently controlled electron-beam matching on staged plasma accelerators.

\section{EMITTANCE GROWTH BY BETATRON DECOHERENCE: THEORY}

The transverse trace-space emittance [17],

$$
\epsilon=\sqrt{\left\langle x^{2}\right\rangle\left\langle x^{\prime 2}\right\rangle-\left\langle x x^{\prime}\right\rangle^{2}}
$$

is a figure of merit for the transverse beam quality, where $x$ is the transverse particle position, $x^{\prime}=p_{x} / p_{\underline{z}}$ is the slope of a particle trajectory, and $\left\langle Y^{k}\right\rangle=\sum_{i}^{N}\left(Y_{i}-\bar{Y}\right)^{k} / N$ the $k$ th central moment of a discrete variable $Y$. The ratio of the transverse momentum $p_{x}$ over the longitudinal momentum $p_{z}$ is damped adiabatically when particles gain 
longitudinal momentum during the acceleration process. To compensate for this effect, the normalized transverse trace-space emittance $\epsilon_{n}=\epsilon \bar{p}_{z} / m_{e} c$ is introduced, with $\bar{p}_{z}, m_{e}$, and $c$ being the particle averaged longitudinal momentum, the electron rest mass, and the speed of light, respectively.

We consider an electron bunch with transverse properties defined by the emittance $\epsilon$ and the Courant-Snyder [18] parameters

$$
\beta=\frac{\left\langle x^{2}\right\rangle}{\epsilon}, \quad \gamma=\frac{\left\langle x^{\prime 2}\right\rangle}{\epsilon}, \quad \alpha=-\frac{\left\langle x x^{\prime}\right\rangle}{\epsilon} .
$$

The beta function is a measure for the beam size and for the betatron length, gamma is a measure for the spread in the particle slopes, and alpha represents the correlation between $x$ and $x^{\prime}$. Combining Eqs. (1) and (2) yields the relation between these parameters, $\beta \gamma=1+\alpha^{2}$.

In the following we assume that the bunch propagates collinear and with a defined temporal offset with respect to a laser pulse on the laser propagation axis. The laser pulse with normalized vector potential $a_{0}$ is focused onto a gas target, ionizes the gas and simultaneously excites plasma waves that carry large amplitude wakefields. Experiments with externally injected electron bunches should be designed such that the laser drives linear $\left(a_{0} \ll 1\right)$ or quasilinear plasma waves $\left(a_{0} \sim 1\right)$ to inhibit self-injection of plasma electrons [19]. The formulation within the scope of this work describes only this regime and is not valid for the highly nonlinear or blowout regime. Since a nonlinear radial dependence of the fields causes emittance growth, the driving laser pulse must have a "parabolalike" radial intensity dependence near axis, $a^{2}(r) \sim 1-\left(r / r_{s}\right)^{2}$, to guarantee for linearly focusing fields in the quasilinear regime. A Gaussian envelope of the laser driver,

$$
a^{2}(\xi, r)=a_{0}^{2} \exp \left(-\frac{\left(\xi-\xi_{l}\right)^{2}}{L^{2}}\right) \exp \left(-\frac{2 r^{2}}{r_{s}^{2}}\right),
$$

complying with this constraint is assumed, where $\xi=$ $z-v_{g} t$ is the comoving variable, $v_{g}$ is the group velocity of the laser, $r_{s}$ is the laser spot size, and $L$ the length of the pulse. The longitudinal electric wakefield component for a resonantly driven plasma wave $\left(L=\sqrt{2} / k_{p}\right)$ for positions behind the laser pulse $\left(\xi-\xi_{l}\right)^{2} \gg L^{2}$ is then given by [20]

$$
\frac{E_{z}}{E_{0}} \simeq \sqrt{\frac{\pi}{2 e}} a_{0}^{2} \exp \left(-\frac{2 r^{2}}{r_{s}^{2}}\right) \cos \left[k_{p}\left(\xi-\xi_{l}\right)\right] .
$$

Here $k_{p}=\omega_{p} / c$ is the plasma wave number, $\omega_{p}$ is the plasma frequency, and $E_{0}$ is the cold nonrelativistic wave breaking field [21]. Moreover, the radial wakefield $E_{r}-c B_{\theta}$ acting on a relativistic, charged particle can be deduced using Maxwell's equations and assuming cylindrical symmetry. This yields for the transverse fields near the axis [22],

$$
\frac{E_{r}-c B_{\theta}}{E_{0}} \simeq-K^{2} k_{p} r \sin \left[k_{p}\left(\xi-\xi_{l}\right)\right],
$$

where $K=\sqrt[4]{8 \pi / e} a_{0} /\left(k_{p} r_{s}\right)$. For simplicity we will use $k_{p} \xi_{l}=\pi$ in the following, such that maximum accelerating field and the zero crossing of the focusing field are located at $\xi=0$. The aim in external injection is to place electron bunches with a length much shorter than the plasma wavelength and a transverse extent much smaller than the laser spot size into the phase region of the wake which is both focusing and accelerating. While being accelerated, the individual particles perform transverse betatron oscillations with a betatron frequency of

$$
\omega_{\beta}^{2}=\frac{K^{2} \omega_{p}^{2}}{\gamma_{r}} \sin \left(k_{p} \xi\right)
$$

where $\gamma_{r}$ is the Lorentz factor. Because of the particle oscillations, the ellipse with area $\pi \epsilon$, defined by the Courant-Snyder parameters [18],

$$
\gamma x^{2}+2 \alpha x x^{\prime}+\beta x^{\prime 2}=\epsilon,
$$

rotates according to the single-particle trajectories in trace space, which are given by

$$
x^{2} / \beta_{m}+\beta_{m} x^{\prime 2}=\text { const, }
$$

where $\beta_{m}$ is deduced from the equation of motion,

$$
\beta_{m}=\frac{\bar{p}_{z}}{m_{e} \gamma_{r} \omega_{\beta}}
$$

Since the betatron frequency [Eq. (6)] is $\xi$-dependent and additionally the electron bunch may have an energy chirp, the individual longitudinal slices of a finite-length bunch oscillate at different frequencies which leads to a $\xi$-dependence of the betatron-oscillation phase, and ultimately to complete decoherence during the acceleration process. This effect is illustrated in Fig. 1, where the tracespace ellipses of longitudinal bunch slices from a PIC simulation are shown for different $z$ positions. The interval $\left[\bar{z}-3 \sigma_{z}, \bar{z}+3 \sigma_{z}\right]$, where $\sigma_{z}=\sqrt{\left\langle z^{2}\right\rangle}$ is the rms bunch

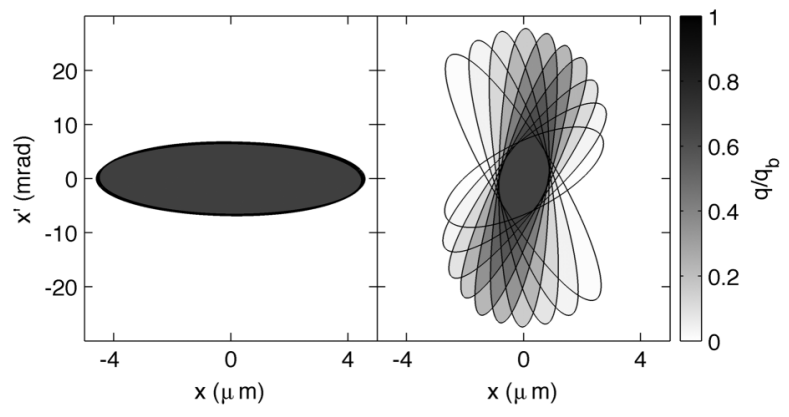

FIG. 1. Ellipses representing bunch slices from PIC simulation C2 (see below) at position $z=-0.03 \mathrm{~mm}$ (left) and $z=$ $1.06 \mathrm{~mm}$ (right). The gray scale of the ellipse was chosen according to the ratio of the charge in a slice $q$ and total bunch charge $q_{b}$. 
length, was divided equidistantly into 10 slices and ellipse parameters for each slice particle subsets were calculated with Eqs. (1) and (2). Slice ellipses develop a tilt with respect to each other which increases the projected area and hence causes growth of the projected emittance whereas the charge-weighted sum over the emittance of the slices, called sliced emittance, does not increase owing to this effect. Emittance growth due to slice-ellipse rotation is theoretically reversible [23]. However, recovering the emittance by further rotation in a subsequent plasma stage requires a transformation $\left(x, x^{\prime}\right) \rightarrow\left(\mp x, \pm x^{\prime}\right)$ in between stages which is not symplectic and hence not realizable by any combination of beam optics.

It should be noted that, for bunches with no significant energy chirp, the betatron decoherence caused by finite energy spread increases the sliced emittance and the projected emittance in the same way. This can be explained by the fact that the decoherence due to energy spread emerges within each slice, in contrast to the decoherence owing to finite bunch length, for which the betatron phase of the various slices decohere with respect to each other. However, complete decoherence generates the same growth of projected emittance in both cases.

Nonetheless, emittance growth from betatron decoherence can be suppressed by matching the transverse properties of the electron beam to the intrinsic electron-betatron motion in the plasma wake. Expressing the matching conditions in terms of the Courant-Snyder parameters in the relativistic limit gives

$$
\beta_{m} \simeq \frac{c}{\omega_{\beta}}, \quad \gamma_{m}=\frac{1}{\beta_{m}} \simeq \frac{\omega_{\beta}}{c}, \quad \alpha_{m}=0,
$$

so that particle oscillations follow the ellipse defined by the Courant-Snyder parameters of the beam. Once a bunch is matched, adiabatic changes of the betatron frequency, that is changes on time scales $T \gg 1 / \omega_{\beta}$ or equivalently on length scales $L \gg c / \omega_{\beta}$, will not degenerate the beam quality.

We transform the trace-space coordinate system such that particles have a circular trajectory to estimate the emittance growth caused by the decoherence of betatron oscillations,

$$
\begin{gathered}
x^{*}=x / \sqrt{\beta_{m}}, \quad x^{\prime *}=x^{\prime} \sqrt{\beta_{m}}, \\
\gamma^{*}=\gamma \beta_{m}, \quad \beta^{*}=\beta / \beta_{m}, \quad \alpha^{*}=\alpha .
\end{gathered}
$$

The emittance [Eq. (1)] is invariant under this transformation. Total phase decoherence implies that the characteristic ellipse of the bunch evolves into a circle in transformed trace-space coordinates with a radius given by the initial standard deviations along the semimajor and semiminor axes. The emittance after total phase mixing can therefore be estimated by

$$
\epsilon_{\mathrm{fin}}=\left\langle x_{r o t}^{2}\right\rangle=\left\langle\frac{x_{a}^{2}+x_{b}^{2}}{2}\right\rangle=\frac{a^{2}}{2}+\frac{b^{2}}{2} .
$$

Here $a$ and $b$ are the semimajor and the semiminor axes of the bunch ellipse in the transformed system prior to phase decoherence:

$$
\begin{aligned}
& a^{2}=\frac{\epsilon_{\text {init }}}{2}\left[\left(\gamma^{*}+\beta^{*}\right)+\sqrt{\left(\gamma^{*}+\beta^{*}\right)^{2}-4}\right], \\
& b^{2}=\frac{\epsilon_{\text {init }}}{2}\left[\left(\gamma^{*}+\beta^{*}\right)-\sqrt{\left(\gamma^{*}+\beta^{*}\right)^{2}-4}\right] .
\end{aligned}
$$

This yields the final normalized emittance for complete decoherence:

$$
\epsilon_{n, \text { fin }}=\frac{\epsilon_{n, \text { init }}}{2}\left(\frac{1+\alpha^{2}}{\beta^{*}}+\beta^{*}\right) .
$$

\section{COMPARISON TO PIC SIMULATIONS}

We compare our model to full 3D PIC simulations using the code OSIRIS [24]. Electron bunches with a low relative energy spread of $\Delta E / E=0.0065$ and an initial normalized emittance of $\epsilon_{n, \text { init }}=0.3 \mu \mathrm{m}$ propagate at a defined temporal offset collinear to a $5 \mathrm{~J}, 25$ fs FWHM length, $800 \mathrm{~nm}$ central wavelength, $a_{0}=1.8$ laser pulse on the laser axis. The Rayleigh length of the laser is long compared to the betatron length and the FWHM intensity waist of the pulse is $50 \mu \mathrm{m}$, where the transverse profile is a Gaussian and the temporal profile is a symmetric polynomial. The dimensions of the copropagating simulation box in the longitudinal and transverse directions are $118 \times$ $336 \times 336 \mu \mathrm{m}^{3}, 4620 \times 250 \times 250$ cells with 1 particle per cell for the plasma and a total number of $\sim 5 \times 10^{5}$ beam particles. The charge was deposited using a quadratic interpolation scheme. Electron beams have a charge of $1 \mathrm{pC}$, mean energy of $5 \mathrm{MeV}$, and an rms bunch length of $\sigma_{z}=3 \mu \mathrm{m}$ resembling realistic parameters obtainable from conventional electron guns. The charge distribution of the bunch in focus is a Gaussian in space and momentum space. We chose the charge sufficiently low to neglect space-charge forces as well as beam loading, the energy low enough to see the betatron-phase mixing effect within the first few millimeters, and the bunch length much shorter than the plasma wavelength. The bunches are injected with a temporal offset of 120 fs with respect to the peak of the laser pulse envelope to allow for optimum acceleration in the focusing phase of the plasma wave. The plasma target has a flattop longitudinal profile with an electron density of $n_{0}=10^{17} \mathrm{~cm}^{-3}$. The rising edge at position $z_{0}=0$ was chosen short compared to the plasma wavelength to simplify the matching conditions. The falling edge is at position $240 c / \omega_{p} \simeq 4.0 \mathrm{~mm}$.

We compare PIC simulations with different sets of Courant-Snyder parameters that result from different focusing geometries of the same electron beam: (i) matched 
case $(\mathrm{CM})$ - beam with matched Courant-Snyder parameters, $\beta_{\mathrm{CM}}\left(z_{0}\right)=\beta_{m}, \alpha_{\mathrm{CM}}\left(z_{0}\right)=\alpha_{m}=0$; (ii) mismatched case $(\mathrm{C} 1)$ - beam with matched beta function at focus $\beta_{\mathrm{CM}}\left(z_{f, \mathrm{C} 1}\right)=\beta_{m}$ but with mismatched focal position $z_{f, \mathrm{C} 1} \neq z_{0} \rightarrow \alpha_{\mathrm{C} 1}\left(z_{0}\right) \neq 0$; (iii) mismatched case (C2)— beam with mismatched beta function $\beta_{\mathrm{C} 2}\left(z_{0}\right) \neq \beta_{m}$ but matched focal position $z_{f, \mathrm{C} 2}=z_{0} \rightarrow \alpha_{\mathrm{C} 2}\left(z_{0}\right)=0$. For the matched case $(\mathrm{CM})$, the focal position of the bunch $z_{f, \mathrm{CM}}$, must be positioned at $z_{0}$ to satisfy $\alpha_{\mathrm{CM}}\left(z_{0}\right)=0$ in the matching conditions (10). We analyzed the slope of the transverse force at the position where the bunch is supposed to be injected and used Eqs. (6) and (9) to find the matching beta function $\beta_{m}=0.126 \mathrm{~mm}$. This corresponds to an rms beam size of $1.97 \mu \mathrm{m}$ for $\epsilon_{n, \text { init }}=0.3 \mu \mathrm{m}$.

In simulation (C1), the focus is at $z_{f, \mathrm{C} 1}=-20 c / \omega_{p} \simeq$ $-0.33 \mathrm{~mm}$. During the initial vacuum propagation the Courant-Snyder parameters evolve according to the formulas for their evolution in a free drift, neglecting spacecharge forces [18],

$$
\begin{gathered}
\alpha(z)=\frac{z_{f}-z}{\beta_{f}}, \quad \beta(z)=\beta_{f}+\frac{\left(z-z_{f}\right)^{2}}{\beta_{f}}, \\
\gamma(z)=\frac{1}{\beta_{f}},
\end{gathered}
$$

where $\beta_{f}$ is the beta function at focus $z_{f}$. The beta and gamma functions at $z_{0}$ in the PIC simulation are $\beta_{\mathrm{C} 1,0}=$ $1.026 \mathrm{~mm}$ and $\gamma_{\mathrm{C} 1,0}=7.937 \mathrm{~mm}^{-1}$. For case $(\mathrm{C} 2)$ the beta and gamma function at $z_{0}$ are $\beta_{\mathrm{C} 2,0}=0.678 \mathrm{~mm}$, $\gamma_{\mathrm{C} 2,0}=1.476 \mathrm{~mm}^{-1}$. Figure 2 depicts the evolution of the Courant-Snyder parameters during acceleration for the three mentioned cases. The evolution of the alpha

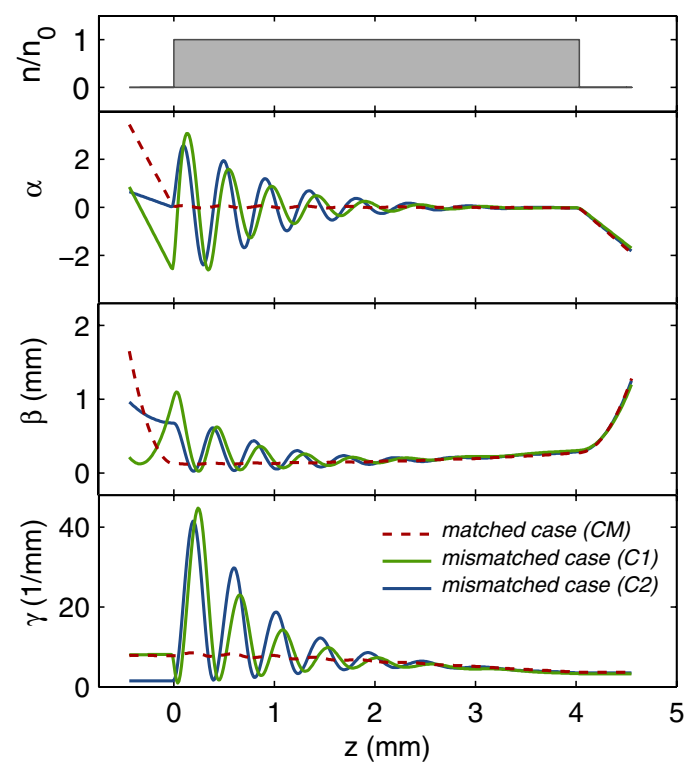

FIG. 2. Plasma density profile and evolution of the Courant-Snyder parameters for the three considered cases in PIC simulations. parameter shows that bunches in simulations $(\mathrm{CM})$ and (C2) are focused to position $z_{0}$ whereas $\alpha_{\mathrm{C} 1}$ crosses zero before $z_{0}$ and the bunch is defocused at position $z_{0}$. This is also indicated by the evolution of the beta parameter. Its parabola vertices (at focus) for cases (CM) and (C2) are situated at $z_{0}$ in contrast to case $(\mathrm{C} 1)$, for which the vertex is in front of the plasma rising edge. The gamma function of (C1) initially equals $\gamma_{\mathrm{CM}}\left(z_{0}\right)$ while $\gamma_{\mathrm{C} 2}$ is not matched. If matched, the bunch ellipse will not oscillate after injection and $\alpha$ will remain zero during the acceleration process as observed for simulation (CM), whereas the alpha parameters in the cases $(\mathrm{C} 1)$ and $(\mathrm{C} 2)$ oscillate around zero. Owing to relativistic mass gain, the beta function increases adiabatically and the gamma function decreases accordingly. The $\alpha, \beta, \gamma$ curves of (C1) and (C2) all approach the matched case (CM) by the cost of emittance growth during betatron-phase mixing as can be seen by comparison of Figs. 2 and 3. We want to emphasize that the emittance in the matched case $(\mathrm{CM})$ did not grow significantly despite the fact that the bunch slipped back substantially with respect to the plasma wave because of the low injection energy. This is because the phase slippage occurs adiabatically and does not disrupt the matching conditions.

The betatron phase is completely mixed at $z \approx 2.5 \mathrm{~mm}$ and emittance growth is saturated at that position in good agreement with expectations [confer Eq. (18) below]. The emittance in the matched case $(\mathrm{CM})$ grows marginally compared to the nonmatched cases. After exiting the plasma target and the beam being at a waist $(\alpha=0)$, the Courant-Snyder parameters evolve again according to Eq. (17).

We now compare these numerical results on the emittance evolution with the above derived analytic theory. The normalized emittance at the plasma exit in the PIC simulation is $\epsilon_{n, \mathrm{C} 1}=1.360 \mu \mathrm{m}$ for case $(\mathrm{C} 1)$ and $\epsilon_{n, \mathrm{C} 2}=$ 0.830 for case (C2). Using formula (16) we find $\epsilon_{n, \mathrm{fin}, \mathrm{C} 1}=$ $1.371 \mu \mathrm{m}$ and $\epsilon_{n, \mathrm{fin}, \mathrm{C} 2}=0.835 \mu \mathrm{m}$. Thus, the theory

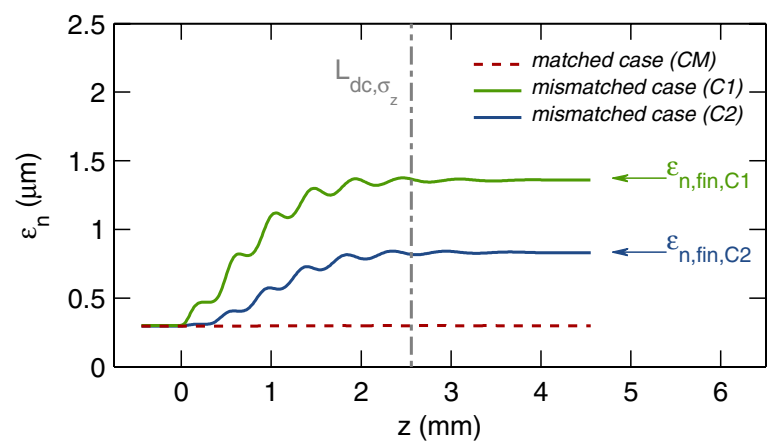

FIG. 3. Evolution of the normalized emittance $\epsilon_{n}$ in PIC simulations for the three considered cases. Arrows show the analytic predictions of the emittance growth. The betatrondecoherence length for the injection phase in the simulations $k_{p} \xi_{0}=1.00$ relative to position $z_{0}$ is indicated by the dashdotted line. 
yields close estimates accurate to within $1 \%$ for emittance growth due to betatron decoherence.

Emittance generation in the PIC calculations by insufficient spatial resolution or by numerical heating effects [25] was shown to be negligible by comparison of the presented simulations to simulations with higher resolution and higher charge-interpolation schemes. The transverse bunch extent was small compared to the laser spot size such that the nonlinearity of the focusing fields at radii on the order of the laser spot size did not cause emittance growth. Comparison of transverse emittance in and perpendicular to the polarization plane ruled out laser heating as an emittance generator. Furthermore, bunch charge densities sufficiently low to neglect space charge and beam loading effects were used.

\section{CONSEQUENCES FOR STAGED ACCELERATION}

The consequences of the presented calculations for staged acceleration are discussed in the following. First, we derive the propagation distance during which the betatron phase of a bunch becomes mixed from finite bunch length. An electron bunch with rms length $k_{p} \sigma_{z} \ll 1$, negligible energy spread, and a charge well below the beam loading limit is considered to find an approximation for this distance. Assuming the bunch is injected and fixed to a phase $\xi_{0}$ in a quasilinear plasma wave, we find an expression for the complete decoherence length caused by finite bunch length,

$$
L_{d c, \sigma_{z}} \simeq \frac{2 \pi c}{\omega_{\beta}\left(\xi_{0}\right) k_{p} \sigma_{z}} \tan \left(k_{p} \xi_{0}\right),
$$

for $\sigma_{z}<\xi_{0}<\left(\lambda_{p} / 4-\sigma_{z}\right)$. The decoherence length from finite energy spread $\sigma_{\gamma}$ is given by (cf. [16]) $L_{d c, \sigma_{\gamma}} \simeq$ $2 \pi c\left\langle\gamma_{r}\right\rangle /\left(\omega_{\beta} \sigma_{\gamma}\right)$. Comparing this expression with Eq. (18) suggests that finite bunch length as a generator of betatron decoherence is dominant over finite energy spread if $k_{p} \sigma_{z} / \tan \left(k_{p} \xi_{0}\right)>\sigma_{\gamma} /\left\langle\gamma_{r}\right\rangle$ holds and vice versa. To ensure for efficient acceleration, the bunch must be injected (and at best fixed, e.g., as described in [26]) close to the maximum accelerating field, i.e. $k_{p} \xi_{0} \leq \pi / 4$, such that $\tan \left(k_{p} \xi_{0}\right) \leq 1$. Thus, for energy spreads as demanded for typical applications [10], the effect from finite length can generally be assumed to be dominant over the effect by finite energy spread. Nevertheless, energy spread and bunch length are strongly interrelated in wakefield and vacuum propagation and both need to be minimized to ensure for good beam quality.

The assumption $k_{p} \xi_{0}<\pi / 4$ simplifies Eq. (18) to $k_{p} L_{d c, \sigma_{z}} \leq 4.28 \sqrt{\gamma_{r}} k_{p} r_{s} /\left(a_{0} k_{p} \sigma_{z}\right)$. The betatron phase is thus completely mixed within the length of a stage, given by the depletion length [27] $k_{p} L_{p d} \simeq 17.4 \lambda_{p}^{2} /\left(\lambda_{l}^{2} a_{0}^{2}\right)$, where $\lambda_{l}$ is the laser wavelength, as long as $k_{p} \sigma_{z} \lambda_{p}^{2} /\left(\sqrt{\gamma_{r}} k_{p} r_{s} a_{0} \lambda_{l}^{2}\right) \gtrsim 0.25$ holds. If we assume $k_{p} \sigma_{z}=0.1, \lambda_{p} / \lambda_{l}=100, a_{0}=2$, and $r_{s}=\lambda_{p} / 2$, parameters which are close to those proposed for a future plasma-based collider design [11], we find this being the case for beam energies of $\lesssim 250 \mathrm{GeV}$. However, the effect of emittance growth by mismatch of the beta function and partial phase mixing is relevant as long as $L_{d c, \sigma_{z}}$ does not significantly exceed the length of an accelerating stage.

The above derived model is now applied to staged plasma acceleration concepts in which no electron-beam optics are used in between stages to minimize the coupling distance $L_{c}$, which contributes critically to the total length of a multistage accelerator [11]. In the following we assume plasma stages longer than the betatron-decoherence length $L_{d c, \sigma_{z}}$, beams thus having a matched beta function $\beta_{m}^{i}=$ $\beta_{f}^{i}$ and being in waist when exiting stage $i$, and subsequently performing a free drift with length $L_{c}$ between stages $i$ and $i+1$. Using an approximation for the betatron frequency $\omega_{\beta} \simeq a_{0} \omega_{p} /\left(k_{p} r_{s} \sqrt{\gamma_{r}}\right)$ and assuming $\beta_{f}^{i}=$ $\beta_{m}^{i+1}$ together with Eqs. (10), (12), (16), and (17) yields

$$
\epsilon_{n, \text { in }} \simeq \epsilon_{n, \text { init }}\left(1+\frac{\eta^{2}}{2 \gamma_{r}}\right)
$$

after complete betatron-phase mixing with $\epsilon_{n \text {,init }}$ and $\epsilon_{n \text {,fin }}$ being the emittance values before and after transmission through stage $i+1$, respectively, and $\eta=a_{0} k_{p} L_{c} /\left(k_{p} r_{s}\right)$. When assuming the use of plasma-based laser mirrors $[28,29]$ to minimize $k_{p} L_{c}$ to values of $\sim 10^{4}$, we find a per-stage emittance increase by a factor $\geq 11$ for electron beams with energies $\leq 500 \mathrm{GeV}$, for $a_{0}=2$ and $r_{s}=$ $\lambda_{p} / 2$, i.e., $\eta \simeq 6.4 \times 10^{3}$, rendering such beams unusable for applications after multiple stages. The per-stage emittance growth as a function of beam energy, depicted in Fig. 4, suggests that the emittance growth per stage becomes insignificant for beam energies $>10 \mathrm{GeV}$ only if $\eta<10^{2}$. With plasma-based and conventional laser-focusing technology this is attainable only if a low laser amplitude $a_{0} \ll 1$, a large spot size $k_{p} r_{s} \gg 1$, or a nonfundamental transverse Gaussian mode with a low transverse intensity gradient are utilized. Since the accelerating field in the wake

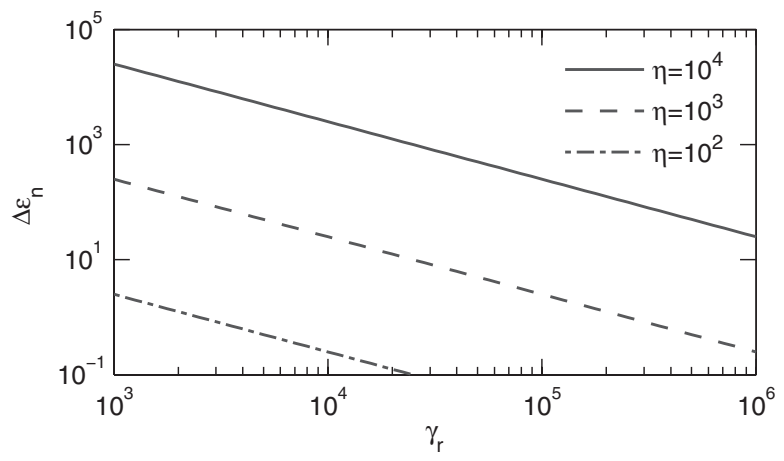

FIG. 4. Relative per-stage emittance growth $\Delta \epsilon_{n}=$ $\epsilon_{n, \text { fin }} / \epsilon_{n \text {,init }}-1$ according to Eq. (19) as a function of beam energy for different values of $\eta=a_{0} k_{p} L_{c} /\left(k_{p} r_{s}\right)$. 
(4) is proportional to $a_{0}^{2}$ and the required laser power increases with $r_{s}^{2}$, maintaining a value of $\eta<10^{2}$ would result in highly inefficient acceleration for pure Gaussian laser modes.

The assumption $\beta_{f}^{i}=\beta_{m}^{i+1}$ for the derivation of Eq. (19) is contributed to the fact that sharp vacuum-plasma interfaces are considered in this study. Another option constitutes the investigation of smooth transitions, much longer than the local betatron frequency in order to match the electron beam adiabatically into the plasma wave, as proposed in earlier works $[13,15]$. This would imply the beta function of the bunch when emerging from stage $i$ to be smaller than the beta function in the subsequent stage $i+1$ before being adiabatically matched, $\beta_{f}^{i}<\hat{\beta}_{m}^{i+1}$, thus reducing emittance growth after a free drift compared to the sharp interface case [cf. derivation of Eq. (19)]. In addition to matching beams adiabatically, we propose to adiabatically cool beams transversely by employing plasma-tovacuum transitions much longer than the local betatron wavelength [30], hence adiabatically increasing the beam spot size $\beta_{m}^{i}<\hat{\beta}_{f}^{i}$ immediately downstream of stage $i$. It should be noted that Eq. (16) implies that adiabatic matching or cooling without additional refocusing cannot inhibit emittance growth completely, since the beam is out of waist $(\alpha \neq 0)$ before entering the subsequent stage. Bunches must thus be refocused to eliminate emittance growth, while adiabatic matching eases the focusing requirements by increasing the beta functions to which beams need to be matched. Further research is necessary to prove the feasibility, stability, and capability of emittance suppression by the aforementioned adiabatic matching or cooling techniques.

\section{SUMMARY AND CONCLUSION}

The present study shows quantitatively that the emittance of an electron beam in an external-injection scheme, as necessary for staging, grows even for ideal laser-bunch alignment (stochastic errors on the alignment have been studied, e.g., in [31]), if the beam parameters are not matched to the intrinsic betatron length in the plasma wake. If matched, the emittance remains constant during the acceleration process within $1 \%$ whereas the emittance of nonmatched beams may increase by orders of magnitude depending on the degree of mismatch.

As demonstrated in the discussed PIC simulations, matched beams stay matched if changes of the beam or wakefield properties occur adiabatically. Since the betatron frequency decreases for higher beam energies, changes of the wakefield amplitude, e.g., laser spot size oscillations [32] might eventually occur on time scales on the order of the inverse betatron frequency, thus disrupting the matching conditions. This, however, is subject to further studies.

The challenge of matching electron beams into plasma waves arises from the fact that the generated focusing fields are comparable in their order of magnitude to the accelerating fields, and the corresponding matched beta function is therefore small. Assmann and Yokoya thus suggested [15] to decouple the amplitude of transverse and longitudinal fields by using transversely tailored drivers to increase the matched beta function. For laser drivers this can be achieved by combinations of higher order Laguerre-Gaussian modes [33] which provide a focusing field with reduced linear radial dependence near axis and hence an increased matched beta function of the injected beam. Application of this technique is equivalent to utilization of an increased spot size $r_{s}$ in Eq. (19) and allows for a reduction, but not for a complete suppression of the emittance growth of unmatched beams after free drifts. In addition, beating of the transverse modes needs to be mitigated to enable a stable focusing channel [33].

An earlier analysis [34] showed that the focusing phase of the wakefield in plasma channels shifts with respect to the accelerating phase such that the phases may entirely overlap in later wave periods and the maxima of transverse and longitudinal component thus being located at the same comoving position. Given a high degree of control over absolute density and transverse density gradient in a plasma channel, this effect offers the possibility of reducing emittance growth from finite bunch length if electron bunches are being placed and fixed in this comoving region and the decoherence length from finite bunch length Eq. (18) hence tending to infinity. Albeit reducing emittance growth from finite bunch length, this method cannot mitigate emittance degradation by finite energy spread of unmatched electron bunches.

In conclusion, we find that refocusing of electron beams is inevitable to efficiently suppress beam-quality degeneration due to betatron-phase decoherence. It may be inferred that this degeneration is relevant for electron energies up to the TeV-energy frontier. These findings will have an essential impact on the design and total length of multistage laser-wakefield accelerators since electron-beam optics, significantly longer than each plasma stage, if based on today's technology, are necessary in between two stages in order to avoid substantial emittance aggregation.

\section{ACKNOWLEDGMENTS}

This project was funded by the Helmholtz Alliance "Physics at the Terascale". We thank the OSIRIS consortium (IST/UCLA) for access to the OSIRIS code. Special thanks for support go to J. Vieira and R. Fonseca. Furthermore, we acknowledge the grant of computing time by the Jülich Supercomputing Centre on JUGENE under Project No. HHH09. We would like to thank DESY IT for their support concerning simulations and data storage at DESY. Finally, we thank C. Behrens for fruitful discussions. 
[1] S. P. D. Mangles et al., Nature (London) 431, 535 (2004).

[2] C. G. R. Geddes, Cs. Toth, J. van Tilborg, E. Esarey, C. B. Schroeder, D. Bruhwiler, C. Nieter, J. Cary, and W. P. Leemans, Nature (London) 431, 538 (2004).

[3] J. Faure, Y. Glinec, A. Pukhov, S. Kiselev, S. Gordienko, E. Lefebvre, J.-P. Rousseau, F. Burgy, and V. Malka, Nature (London) 431, 541 (2004).

[4] W. P. Leemans, B. Nagler, A. J. Gonsalves, Cs. Tóth, K. Nakamura, C. G. R. Geddes, E. Esarey, C. B. Schroeder, and S. M. Hooker, Nat. Phys. 2, 696 (2006).

[5] C. G. R. Geddes, K. Nakamura, G. Plateau, Cs. Toth, E. Cormier-Michel, E. Esarey, C. Schroeder, J. Cary, and W. Leemans, Phys. Rev. Lett. 100, 215004 (2008).

[6] J. Osterhoff et al., Phys. Rev. Lett. 101, 085002 (2008).

[7] A. J. Gonsalves et al., Nat. Phys. 7, 862 (2011).

[8] M. Fuchs et al., Nat. Phys. 5, 826 (2009).

[9] S. Kneip et al., Nat. Phys. 6, 980 (2010).

[10] W. Leemans and E. Esarey, Phys. Today 62, 44 (2009).

[11] C. B. Schroeder, E. Esarey, C. Geddes, C. Benedetti, and W. Leemans, Phys. Rev. ST Accel. Beams 13, 101301 (2010).

[12] E. Esarey et al., AIP Conf. Proc. 737, 578 (2004).

[13] K.A. Marsh et al., in Proceedings of the Particle Accelerator Conference, Knoxville, TN, 2005 (IEEE, Piscataway, NJ, 2005), pp. 2702-2704.

[14] A. G. Khachatryan, A. Irman, F. van Goor, and K.-J. Boller, Phys. Rev. ST Accel. Beams 10, 121301 (2007).

[15] R. Assmann and K. Yokoya, Nucl. Instrum. Methods Phys. Res., Sect. A 410, 544 (1998).

[16] P. Michel, C. Schroeder, B. Shadwick, E. Esarey, and W. Leemans, Phys. Rev. E 74, 026501 (2006).

[17] K. Floettmann, Phys. Rev. ST Accel. Beams 6, 034202 (2003).

[18] E. Courant and H. Snyder, Ann. Phys. (N.Y.) 3, 1 (1958).
[19] A. Modena et al., Nature (London) 377, 606 (1995).

[20] L. M. Gorbunov and V. I. Kirsanov, Sov. Phys. JETP 66, 290 (1987) [Zh. Eksp. Teor. Fiz. 93, 509 (1987)].

[21] J. M. Dawson, Phys. Rev. 113, 383 (1959).

[22] R. Keinigs and M. E. Jones, Phys. Fluids 30, 252 (1987).

[23] P. G. O’Shea, Phys. Rev. E 57, 1081 (1998).

[24] R. A. Fonseca et al., Lect. Notes Comput. Sci. 2331, 342 (2002); R. A. Fonseca, S. F. Martins, L. O. Silva, J. W. Tonge, F.S. Tsung, and W. B. Mori, Plasma Phys. Controlled Fusion 50, 124034 (2008).

[25] E. Cormier-Michel, B. Shadwick, C. Geddes, E. Esarey, C. Schroeder, and W. Leemans, Phys. Rev. E 78, 016404 (2008).

[26] W. Rittershofer, C. B. Schroeder, E. Esarey, F. J. Gruner, and W. P. Leemans, Phys. Plasmas 17, 063104 (2010).

[27] B. A. Shadwick, C.B. Schroeder, and E. Esarey, Phys. Plasmas 16, 056704 (2009).

[28] D. Panasenko, A. J. Shu, A. Gonsalves, K. Nakamura, N.H. Matlis, Cs. Toth, and W.P. Leemans, J. Appl. Phys. 108, 044913 (2010).

[29] T. Sokollik et al., AIP Conf. Proc. 1299, 233 (2010).

[30] C. M.S. Sears, A. Buck, K. Schmid, J. Mikhailova, F. Krausz, and L. Veisz, Phys. Rev. ST Accel. Beams 13, 092803 (2010).

[31] S. Cheshkov, T. Tajima, W. Horton, and K. Yokoya, Phys. Rev. ST Accel. Beams 3, 071301 (2000).

[32] C. Benedetti, C.B. Schroeder, E. Esarey, and W.P. Leemans, Phys. Plasmas 19, 053101 (2012).

[33] E. Cormier-Michel, E. Esarey, C. Geddes, C. Schroeder, K. Paul, P. Mullowney, J. Cary, and W. Leemans, Phys. Rev. ST Accel. Beams 14, 031303 (2011).

[34] N.E. Andreev, L. M. Gorbunov, V.I. Kirsanov, K. Nakajima, and A. Ogata, Phys. Plasmas 4, 1145 (1997). 